\title{
The effect of redundant stimulus elements on visual discrimination as a function of element heterogeneity, equal discriminability, and position uncertainty'
}

\author{
W. R. GARNER ${ }^{2}$ AND J. H. FLOWERS \\ YALE UNIVERSITY
}

\begin{abstract}
Garner and Lee (1962) showed no gain in visual discrimination accuracy with addition of redundant stimulus elements. Eriksen and Lappin (1965) showed a substantial gain. One experiment reported here indicates that the discrepancy is not due to the fact that the earlier experiment used heterogeneous stimulus elements. $A$ second experiment indicated that the gain in discrimination accuracy does occur when the additional stimulus elements have the same discriminability as the original elements. It also showed that position uncertainty itself has no effect on the gain with redundant elements, but is a convenient procedural device for maintaining fixation and thus equal element discriminability. Three models of perceptual independence fit the data.
\end{abstract}

Garner and Lee (1962) investigated the visual discrimination of patterns of $\mathrm{Xs}$ and $\mathrm{Os}$ presented at short duration and low contrast, and found that as redundant $\mathrm{Xs}$ and/or Os were added to the patterns there was no improvement in discrimination. In fact, there was clear evidence that performance was better if $S$ deliberately avoided use of the redundant elements by attending only to part of the stimulus pattern, thus intentionally excluding perception of the redundant elements.

Eriksen and Lappin (1965a) investigated discrimination of the letters $A, T$, and $U$, presented at short duration, and found that discrimination accuracy improved if more than a single element was presented simultaneously. Thus, their results showed that redundancy improved visual discrimination.

The purpose of the experiments reported here is to determine the reason for the discrepant results from these two experiments. While the two experiments involved basically the same type of visual discrimination task, there were several respects in which they differed, and three of these respects were investigated.

First, stimulus heterogeneity. In the Garner and Lee experiment, four different stimuli of two elements each were used as the minimum, and these were $\mathrm{XX}, \mathrm{XO}, \mathrm{OX}$, or $\mathrm{OO}$. When redundancy was added, either two or seven additional elements were added, and these were always mixtures of Xs and Os. Thus, the redundant elements were not simply repetitions of the same element, but involved two different basic elements. In the Eriksen and Lappin experiment, the three different letters used singly formed the minimum set of stimuli, and when redundant elements were added, the same letter was simply repeated, up to a total of six identical elements. Thus, the failure to obtain increased accuracy of discrimination in the Garner and Lee experiment may have been due to the heterogeneity of the stimulus elements. A recent experiment by Keeley and Doherty (1968) on this problem showed that there was no gain in discrimination accuracy due to redundancy when Landolt rings were the stimulus elements. While this experiment does not bear directly on the question of stimulus heterogeneity, it does demonstrate the importance of the particular stimuli used.

Second, equal discriminability. In the Garner and Lee experiment, the nonredundant stimuli contained two elements placed on a horizontal line. The stimuli containing redundant elements were squares of either $2 \times 2$ or $3 \times 3$ elements. Since visual acuity decieases as retinal position away from the fovea is increased, this arrangement means that the added redundant elements cannot be as discriminable as the two elements used for the nonredundant stimuli, regardless of where $S$ fixates on the stimulus. (See Collins \& Eriksen, 1967, for direct evidence of the effect of visual angle on this type of discrimination task.) In the Eriksen and Lappin experiment, the stimulus elements occurred at six different possible positions on the circumference of a circle, an arrangement that more nearly provides equal discriminability for all stimulus elements, regardless of the number of elements used. Thus, the failure to obtain increased accuracy of discrimination in the Garner and Lee experiment may have been due to this lack of equal discriminability of redundant elements.

Third, position uncertainty. In the Garner and Lee experiment, $S$ always knew how many stimulus elements to expect and in what location, and, furthermore, he was free to look at any part of the stimulus. In the Eriksen and Lappin experiment, a fixation point was used at the center of the circle on whose circumference the stimulus elements occurred, and $\mathrm{S}$ did not know at which or how many of the six positions stimulus elements would occur on a given trial. Lockhead (1965) argued that this position uncertainty was what made the gain due to redundancy possible, and presented data showing that two letters were more discriminable than one only if position was uncertain. Eriksen and Lappin (1965b) argued that position uncertainty was necessary to ensure that $S$ maintained his fixation, since without the position uncertainty $S$ tended to move his eyes in anticipation of the stimulus presentation, thereby using more foveal vision. Thus, Eriksen and Lappin argued that position uncertainty per se was not the critical factor, but rather that the consequent maintenance of equal discriminability for all stimulus elements was the critical factor. Nevertheless, it is possible that position uncertainty per se is responsible for the discrepancy in the two experiments.

\section{EXPERIMENT 1 STIMULUS HETEROGENEITY}

Subjects

Six college students and two staff members served as Ss.

\section{Apparatus and Procedure}

A three-field tachistoscope (Scientific Prototype Model GB) was used. S used binocular viewing at a fixation distance of approximately $40 \mathrm{in.} \mathrm{A} \mathrm{given} \mathrm{trial} \mathrm{was} \mathrm{initiated} \mathrm{by} \mathrm{S's} \mathrm{pressing} \mathrm{a}$ hand-held switch. Prior to the trial $S$ observed a fixation point on an otherwise blank field, with a brightness of approximately $29 \mathrm{ft}-\mathrm{L}$. As soon as $\mathrm{S}$ pressed his switch, the fixation point disappeared and another blank field came on for $100 \mathrm{msec}$ (to prevent interference of the stimulus by the fixation point). This second blank field had a brightness of approximately $24 \mathrm{ft}-\mathrm{L}$. Then the stimulus field ( $5 \mathrm{ft}-\mathrm{L}$ ) was added to this second blank field, in order to provide a low contrast of approximately $21 \%$. The stimulus was exposed for a constant time for each $S$, ranging from 14 to $30 \mathrm{msec}$ for different $\mathrm{Ss}$; these times were selected to 


STIMULUS $\left[\begin{array}{ccccc} & \multicolumn{5}{c}{\text { CONDITION }} \\\right.$\cline { 2 - 5 } & $4 I & 4 M & 2 I & 2 M \\ 8 & \times \times \times & \times 0 & \times \cdot \times & \times \cdot 0 \\ 8 & 0.0 & 0 . \times & 0 \cdot 0 & 0 \cdot \times \\ & 0.0 & \times 0 & & \\ & 83.0 & 83.0 & 79.1 & 82.1\end{array}$

Fig. 1. Stimulus conditions and per cent correct responses for Experiment 1. Each stimulus has either identical (I) or mixed (M) elements. The dot in each stimulus pattern indicates location of the fixation point.

provide approximately $75 \%$ accuracy. When the stimulus field went off, the second blank field was maintained for approximately $620 \mathrm{msec}$ before the first blank field with fixation point returned. After a single trial, $\mathrm{E}$ changed the stimulus and $\mathrm{S}$ initiated the next trial at his option.

\section{Stimulus Conditions}

Each stimulus condition consisted of two alternative stimuli, responded to as $\mathrm{A}$ and $\mathrm{B}$. A card showing the stimuli and correct responses was available for $S$ to inspect at any time. The stimulus elements were $\mathrm{Xs}$ or Os, black on white cards, drawn with India ink. Each $\mathrm{X}$ or $\mathrm{O}$ was always $1 / 8 \mathrm{in}$. high, and the horizontal or vertical distance between centers of stimulus elements was always $1 / 4$ in. These stimulus spacings, at the viewing distance of $40 \mathrm{in}$., gave equivalent visual angles that were slightly less than those used both by Garner and Lee and by Eriksen and Lappin. Four different pairs of stimuli were used, containing either two or four elements, with either mixed or identical elements, as illustrated in Fig. 1.

Four identical elements (4I). A stimulus was either four Xs or four Os arranged in a square, with the fixation point equidistant from all four elements.

Four mixed elements (4M). Each stimulus contained two Xs and two Os, arranged in a square, with diagonal elements being the same. The fixation point was equidistant from all four elements.

Two identical elements (2I). Each stimulus contained either two Xs or two Os, arranged horizontally, with the fixation point midway between the two elements.

Two mixed elements (2M). Each stimulus contained one $\mathrm{X}$ and one $O$, arranged horizontally, with the fixation point midway between the two elements.

This arrangement of stimulus elements is like that used by Garner and Lee, in that when two elements are used, the distance of each element from the fixation point is less than when four elements are used. Thus, this experiment provides a test of the effect of element heterogeneity when equal discriminability of elements is not maintained from the two- to four-element conditions.

It should also be noted that the two-element stimuli are themselves redundant in that only a dichotomous decision was required by $S$. Thus, these experimental conditions strictly speaking provide a test of the effectiveness of additional redundancy rather than minimum redundancy.

Each $S$ was run 100 trials on each condition in a given session, with a Latin square providing counterbalancing of conditions within sessions across Ss. All trials on a given condition were completed before $S$ shifted to another condition, and $S$ was allowed rest between conditions if he wanted. One session lasted about $1.5 \mathrm{~h}$. Data from the first session were used only to provide practice and establish the stimulus duration for each $S$. Then two more sessions were run, no two sessions occurring in the same morning or afternoon. These last two sessions provided 200 trials per condition per $\mathrm{S}$, and provide all data for analysis.

\section{Results}

Per cent correct responses obtained with each of these four conditions are shown in Fig. 1. There are no significant differences between conditions $(F=1.03)$. Values of $d^{\prime}$ were also calculated for each $S$ and these measures show no significant differences either. Condition 2 I appears to give lower accuracy than the others, but the fact that this condition provided the greatest accuracy for three of the eight Ss emphasizes the lack of statistical significance of the result.

Thus, there was no difference due to the use of mixed rather than identical elements. So the discrepancy between the Garner and Lee result and the Eriksen and Lappin result is not due to element heterogeneity.

This result also comple tely confirms the earlier result of Garner and Lee in showing no discrimination gain due to the addition of further redundant elements, even though the present experiment differed from the earlier one by the use of a fixation point. It should be remembered, however, that the use of the fixation point guaranteed that each of the four stimulus elements was farther from the fovea than each of the two stimulus elements, with a resultant lower discriminability. This result then suggests that there might have been a gain in discrimination accuracy by increasing the number of elements if discriminability had not been lowered.

\section{EXPERIMENT 2}

\section{EQUAL DISCRIMINABILITY AND POSITION UNCERTAINTY}

The second experiment was designed to determine whether a discrimination gain with additional redundant elements would occur if all stimulus elements were maintained equidistant from the fixation point to ensure equal discriminability, and also to determine whether position uncertainty had an effect on discriminability other than in its function of maintaining fixation, and thus equal discriminability.

All procedural factors were the same as in Experiment 1. Seven of the eight Ss used in the second experiment had also been used in the first experiment. The stimulus durations used ranged from 15 to 23 msec.

\section{Stimulus Conditions}

Four stimulus conditions were again used, with two alternative stimuli per condition to be reported by $S$. Variations were

\begin{tabular}{|c|c|c|c|c|c|}
\hline & \multicolumn{4}{|c|}{ CONDITION } \\
\hline & & $4 \mathrm{I}$ & $\begin{array}{c}2 \text { I } \\
\text { above }\end{array}$ & $\begin{array}{c}\text { 2I } \\
\text { below }\end{array}$ & $\begin{array}{c}2 \mathrm{I} \\
\text { random }\end{array}$ \\
\hline \multirow{2}{*}{ STIMULUS } & A & & $x_{\cdot} \times$ & & \\
\hline & B & $\begin{array}{l}0.0 \\
0^{\circ}\end{array}$ & 0.0 & & \\
\hline$\%$ CORRE & & 85.1 & 79.2 & 78.7 & 77.4 \\
\hline
\end{tabular}

Fig. 2. Stimulus conditions and per cent correct responses for Experiment 2. Each stimulus has identical elements placed above and below, above, below, or above or below randomly. The dot in each stimulus pattern indicates location of the fixation point. 
number of elements and position uncertainty, but with all elements equidistant from the fixation point, as illustrated in Fig. 2.

Four identical elements (4I). This condition is the same as that used in Experiment 1. Fixation point was again equidistant from all four elements.

Two identical elements above (2I above). Each stimulus contained either two $\mathrm{Xs}$ or two Os, arranged horizontally and above the fixation point in the same location as the top two elements in Condition $4 \mathrm{I}$. S knew in this condition that the stimuli were always above, but was instructed to maintain fixation on the dot.

Two identical elements below (2I below). This condition is the same as the last, except the two elements were placed below the fixation point in the same location as the two lower elements in Condition 4I.

Two identical elements with random location (2I random). The two stimuli contained either two $\mathrm{Xs}$ or two Os, horizontally arranged as in the other conditions, but the location of the stimuli above or below the fixation point occurred randomly. $\mathrm{S}$ did not have to report location, only whether Xs or Os had occurred regardless of location.

\section{Results}

Per cent correct responses obtained with each of these four conditions are shown in Fig. 2. Analysis of variance shows that there is statistical significance to the differences $(F=6.35$, $\mathrm{p}<.01$ ). Condition $4 \mathrm{I}$ gives superior performance to any of the conditions involving only two stimulus elements, and this result is very consistent across $S s$, with seven of the eight $\mathrm{Ss}$ showing best performance with four stimulus elements. Thus, the addition of redundant stimulus elements aids discrimination when the additional elements have equal discriminability.

Further analysis of the results, both parametrically and nonparametrically, fail to show any significance to the small differences obtained between the two conditions with two elements but known position and the condition with two elements but random position. Thus, we have no evidence that position uncertainty per se affects discrimination accuracy. Therefore, this factor is not responsible for any failure to obtain increased discrimination accuracy with the addition of redundant stimulus elements.

\section{DISCUSSION}

There are three points worth brief discussion on these and the preceding data.

\section{Position Uncertainty or Equal Sensitivity?}

This latter result, showing very slight difference in results with two-element stimuli, does not agree with the Lockhead (1965) equivalent comparison for single-element stimuli, nor with Eriksen and Lappin's (1965b) explanation that the use of position uncertainty is necessary in order to prevent premature fixations on the stimulus location rather than on the fixation point. Our results do show a slight improvement when position is known, but it would appear that our Ss were reasonably able to maintain fixation as specifjed by $\mathbf{E}$ even with a known location for the stimulus itself. Possibly our use of a reasonably small visual angle between fixation point and stimulus elements made it easier for our Ss to maintain fixation.

At any rate, this experimental result clarifies the point that the role of position uncertainty is only to guarantee equal sensitivity when performance with two- and four-element stimuli is being compared. Thus, it is simply a convenient procedural device, as Eriksen and Lappin (1965b) in effect argued.

\section{Redundancy vs Foveal Attention}

The fact that the use of a procedure using position uncertainty seems desirable to maintain equal discriminability of all elements in redundant stimuli has interesting implications itself. Garner and Lee (1962) carried out a secondary experiment with sophisticated Ss, asking them to attempt to use all elements of redundant stimuli, or alternatively to attend selectively to enough elements to provide minimal discrimination needs. Their results showed better performance with selective attention, a fact that suggests that if redundant elements are added to a foveal stimulus, no gain in discrimination accuracy would occur. In other words, in this type of discrimination task, with minimally visible stimulus elements, foveal vision is worth more than redundant elements.

\section{Models of Perceptual Independence}

In order to clarify how the organism uses redundant information, we usually invoke the concept of perceptual independence, with the assumption that somehow the organism combines independently obtained information in order to increase the probability of making the correct discriminative response. For this purpose, some model that states what performance ought to be obtained if there is perceptual independence is used as a norm against which to compare actual performance. We shall compare three different normative models, each appropriate to a common measure of performance used in such experiments: probability of correct response, $\mathrm{d}^{\prime}$, and information transmission. For each of these we shall use the data from the 2I random condition in Experiment 2 to predict, according to the appropriate model, performance on the $4 \mathrm{I}$ condition in the same experiment.

Two-state model. When data are described in terms of per cent correct responses, the most appropriate model is one that assumes that the organism is, at any given instant, in one of several states of sensitivity, and that the probability of being in one state for one element is independent of the state the organism is in with respect to the other elements. In a simple form of this model we assume two states of the organism: in one state accuracy is perfect, and in the other it is chance. Thus, the obtained proportion of correct responses ( $\mathrm{p}_{2}$ for the $2 \mathrm{I}$ random condition) is

$$
\mathrm{p}_{2}=\pi+\frac{1}{\mathrm{n}}(1-\pi)
$$

where $\pi$ is the probability that at least one of the two elements occurs in the state of perfect accuracy, and $(1-\pi)$ is the probability of being in the state where chance accuracy occurs; i.e., neither element is in the state of perfect accuracy. $n$ is the number of response alternatives, two in our experiments. To calculate the expected proportion of correct responses for Condition $4 \mathrm{I}\left(\mathrm{p}_{4}^{\prime}\right)$, we solve Eq. 1 for $\pi$, and then apply that value in the following equation, which assumes that the probability of the additional elements being in a given state is independent of the probability that the original elements are in that state:

$$
\mathrm{p}_{4}^{\prime}=\left[1-(1-\pi)^{2}\right]+\frac{1}{\mathrm{n}}(1-\pi)^{2}
$$

The quantity $\left[1-(1-\pi)^{2}\right]$ is the probability that at least one of the four elements occurs in the state of perfect accuracy, and $(1-\pi)^{2}$ is the probability that all four elements occur in the state of chance accuracy.

Values of obtained and predicted per cent correct responses for each $S$ are shown in Table 1 . On the average, this two-s tate model 
Table $\mathbf{~}$

Per Cent Correct Responses for Conditions 21 Random and 41 in Experiment 2. The Predicted Values for Condition 41 are Calculated from Obtained Values for Condition 21 Random and a Two-State Model of Independence

\begin{tabular}{lccc}
\hline$S$ & 21 Random & & 41 \\
\hline 1 & 71.0 & Obtained & Predicted \\
\hline 2 & 72.5 & 82.5 & 83.2 \\
3 & 70.5 & 87.5 & 84.9 \\
4 & 71.0 & 81.0 & 82.6 \\
$5:$ & 82.5 & 79.0 & 83.2 \\
6 & 83.0 & 81.5 & 93.8 \\
7 & 81.5 & 83.5 & 94.2 \\
8 & 87.5 & 94.5 & 93.2 \\
Average & 77.4 & 85.1 & 96.8 \\
\hline
\end{tabular}

Table 2

Values of $d^{\prime}$ for Conditions 21 Random and 41 in Experiment 2. The Predicted Values for Condition 41 are Calculated from Obtained Values for Condition 21 Random and a Euclidean Integration Model of Independence

\begin{tabular}{lccc}
\hline & 21 Random & Obtained & 41 \\
\hline 1 & 1.11 & 1.89 & Predicted \\
2 & 1.22 & 2.32 & 1.57 \\
3 & 1.08 & 1.76 & 1.72 \\
4 & 1.16 & 1.64 & 1.53 \\
5 & 1.87 & 1.83 & 1.64 \\
6 & 2.05 & 2.78 & 2.64 \\
7 & 1.85 & 1.97 & 2.90 \\
8 & 2.31 & 3.23 & 2.62 \\
Average & 1.58 & 2.18 & 3.27 \\
\hline
\end{tabular}

Table 3

Values of Information Transmission in Bits for Conditions 21 Random and 41 in Experiment 2. The Predicted Values for Condition 41 are Calculated from Obtained Values for Condition 21 Random and an Information Model of Independence

\begin{tabular}{lccc} 
& 2I random & & 4I \\
obtained & predicted \\
1 & .132 & .334 & .244 \\
2 & .155 & .459 & .283 \\
3 & .125 & .299 & .232 \\
4 & .138 & .262 & .254 \\
5 & .331 & .315 & .557 \\
6 & .362 & .585 & .601 \\
7 & .318 & .357 & .538 \\
8 & .457 & .695 & .734 \\
Average & .252 & .413 & .430 \\
\hline
\end{tabular}

overpredicts performance on Condition 4I, and also overpredicts for seven of the eight $\mathrm{Ss}$. Thus, this simple two-state model fares only moderately well.

However, Eriksen (1966) has shown that a multistate model tends to predict lower values than the simple two-state model, but a more refined response continuum than we used here is necessary to apply a multistate model. In addition, a simple modification of the two-state model so that the two states are less extreme, leading, for example, to $90 \%$ and $10 \%$ accuracy, rather than $100 \%$ and $0 \%$ accuracy, will improve accuracy of prediction. In fact, for any one $S$, such a model, allowing the solution of one additional parameter from the data, would give a perfect fit, since we would be solving for two parameters with just two data points. What is clear, then, is that at least one and probably several different state models can be shown to fit these data, and thus to justify the assumption of perceptual independence.

A $d^{\prime}$ integration model. A second commonly used measure of discrimination is $\mathrm{d}^{\prime}$ (Green \& Swets, 1966). The model of perceptual independence appropriate with this measure, for a doubling of elements, is quite simple, being

$$
\left(d_{4}^{\prime}\right)^{\prime}=\left(2 d_{2}^{\prime 2}\right)^{1 / 2},
$$

where $d_{2}^{\prime}$ is the measure of discrimination obtained from Condition 2I random, and $\left(\mathrm{d}_{4}^{\prime}\right)^{\prime}$ is the value predicted for Condition 4I. This prediction is based simply on the Pythagorean relation for right triangles.

Values of obtained and predicted $d^{\prime}$ for each $S$ are shown in Table 2 . In this case the average obtained $d^{\prime}$ for Condition $4 I$ is very close to the average predicted value. Furthermose, the model underpredicts for exactly half of the Ss. Thus, we have every reason to accept the assumption of perceptual independence with this model.

An information model. A two-state model is, as its name indicates, a model that assumes the organism to be in varying states, and these are states of sensitivity. The gain in discrimination accuracy occurs because, in effect, the organism has multiple looks, i.e., more chances at correct perception. The $\mathrm{d}^{\prime}$ integration model is basically a process model (set-Garner \& Morton, in press), and assumes that the gain in discrimination accuracy occurs because the organism combines information. Garner and Lee (1962) presented a similar model that predicts the gain in information transmission when the number of stimulus elements is redundantly doubled. It is, of course, appropriate for the third commonly used measure of discrimination, information transmission.

The basic prediction equation is:

$$
\mathrm{IT}_{2}=1 / 2 \log _{2}\left[\mathrm{~V}^{\prime}(\mathrm{S})+1\right]
$$

where $\mathrm{IT}_{2}$ is the obtained information transmission for Condition $2 I$, and $V^{\prime}(S)$ is an hypothetical variance associated with the perceptual process. (See Garner, 1960, for the rationale.) This equation is solved for $V^{\prime}(S)$, and then the prediction for Condition $4 \mathrm{I}\left(\mathrm{IT}_{4}^{\prime}\right)$, with twice as many elements, is:

$$
\mathrm{IT}_{4}^{\prime}=1 / 2 \log _{2}\left[2 \mathrm{~V}^{\prime}(\mathrm{S})+1\right]
$$

Values of obtained and predicted information transmission for each $\mathrm{S}$ are shown in Table 3 . Once again the average obtained information transmission is reasonably close to the average predicted value, and the model underpredicts for exactly half the Ss.

This model is also a process model in that it assumes there to be an integration of information, in this case represented by the doubled $V^{\prime}(S)$ term in Eq. 5. Since it assumes an underlying normal variance process, it is very similar to the $\mathrm{d}^{\prime}$ integration model; thus, it is not surprising that it provides evidence of perceptual independence about as good as that provided by the $\mathrm{d}^{\prime}$ model. However, there is a limitation to the information model with small numbers of stimulus and response alternatives in that the information transmission has a value limited by these numbers of alternatives. This fact tends to produce overprediction for larger values of information transmission, and this systematic bias can be seen in Table 3 . The d' model also has such a limitation when accuracy approaches $100 \%$, but this limitation is much less severe. Thus, the $\mathrm{d}^{\prime}$ measure and model are clearly more appropriate with dichotomous stimuli and responses. On the other hand, the information model is more appropriate with larger numbers of stimuli and responses.

Even though these models do predict quite well, our subjective evidence favors a multistate model, since the stimulus elements seem to be used in the sense of a multiple choice rather than in the sense of combining information. Thus, if two Xs are 
presented, $\mathrm{S}$ does not somehow combine evidence; rather, he makes his decision on the basis of which of the two elements was clearer. And we agree with Eriksen (1966) that a two-state model is too simple, since there are at least three clearly differentiable levels of clarity to the stimulus elements.

Our primary reason for presenting models and showing good fits with data, when we do not feel the models are correct in describing how the organism gains from having redundant information, is to emphasize a point made by Garner and Morton (in press) about the role of models with redundant stimulus experiments. They stated that "it is almost impossible not to find a model which satisfies both the concept of independence and any given experimental result" when correlated stimulus inputs are used. Here we have shown that at least three models can provide very good fits to the data. There is, then, no dearth of models with which to "prove" perceptual independence. This multiplicity of fits of model to data means that simple fitting of data to a model is not sufficient; varied attacks on the problem which more directly test the assumptions of the model are necessary.

\section{REFERENCES}

COLLINS, J. F., \& ERIKSEN, C. W. The perception of multiple simultaneously presented forms as a function of foveal spacing. Perception \& Psychophysics, 1967, 2, 369-373.

ERIKSEN, C. W. Independence of successive inputs and uncorrelated error in visual form perception. Journal of Experimental Psychology, 1966, $72,26-35$

ERIKSEN, C. W., \& LAPPIN, J. S. Internal perceptual system noise and redundancy in simultaneous inputs in form identification. Psy chonomic Science, 1965a, 2, 351-352.

ERIKSEN, C. W., \& LAPPIN, J. S. Reply to Lockhead. Psychonomic Science, $1965 b, 3,366$.

GARNER, W. R. Rating scales, discriminability, and information transmission. Psychological Review, 1960, 67, 343-352.

GARNER, W. R., \& LEE, W. An analysis of redundancy in perceptual discrimination. Perceptual \& Motor Skills, 1962, 15, 367-388.

GARNER, W. R., \& MORTON, J. Perceptual independence: Definitions, models, and experimental paradigms. Psy chological Bulletin, in press.

KEELEY, S. M., \& DOHERTY, M. E. Simultaneous and successive presentations of single-featured and multi-featured visual forms: Implications for the parallel processing hypothesis. Perception \& Psychophysics, 1968, 4, 296-298.

LOCKHEAD, G. R. Repetition redundancy and visual form discrimination. Psychonomic Science, 1965, 3, 319-320.

\section{NOTES}

1. This study was supported by Grant MH 14229 from the National Institute of Mental Health to Yale University. The authors are grateful to John Morton and to Richard L. Gottwald, who helped in the formulation of the problem and the experiments.

2. Department of Psychology, Yale University, New Haven, Connecticut 06510 .

(Accepted for publication March 25, 1969.) 